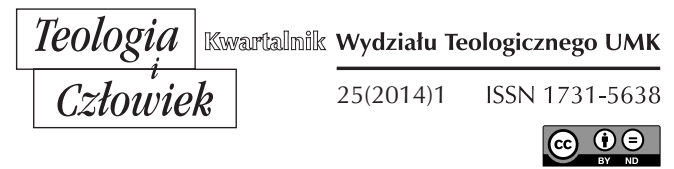

O. DR HAB. ADAM JÓZEF SOBCZYK MSF*

\title{
DIMENSIONE MISTICA DELL'UNIONE CORPOREA DEI CONIUGI CRISTIANI
}

DOI: http://dx.doi.org/10.12775/TiCz.2014.006

Il modo di vedere il matrimonio e la famiglia cambiava lentamente lungo i secoli. Per molti anni, fino alla nascita di Gesù, era un segno della relazione di Dio con il Popolo eletto. Grazie all'Incarnazione del Figlio di Dio ha ricevuto un nuovo significato, ricevendo il suo elemento soprannaturale e diventando un'immagine e un simbolo dell'amore di Cristo alla Chiesa. Dopo la morte del Messia, diventa, come insegna il Concilio Vaticano II, una comunità d'amore (cf. GS 47), in cui i coniugi realizzano e aggiornano l'amore di Dio nel mondo. Un loro compito è il rispecchiare l'amore del Creatore in tutti i luoghi e le dimensioni della vita matrimoniale e familiare (cf. FC 12-13). Questo concerne ugualmente la loro partecipazione alla vita sociale e politica, come il loro intimo rapporto

* Dr hab. Adam Józef Sobczyk - kapłan ze Zgromadzenia Misjonarzy Świętej Rodziny - adiunkt Zakładu Teologii Duchowości UMK w Toruniu. Od 2007 roku członek Międzynarodowej Komisji Formacyjnej Zgromadzenia Misjonarzy Świętej Rodziny, od 2009 roku członek korespondent Polskiego Stowarzyszenia Teologów Duchowości. Wykładowca teologii duchowości, kierownictwa duchowego i poradnictwa rodzin; rekolekcjonista. Zainteresowania naukowe obejmują głównie zagadnienia historii i teologii duchowości ze szczególnym uwzględnieniem duchowości zakonnej oraz duchowości małżeńskiej i rodzinnej. 
interiore. Questa comunità, costruita sull'alleanza santa e indissolubile, mostra il suo carattere nuziale, basato sull'amore di Cristo verso la Chiesa (cf. Ef 5, 25-26), accoglie come lo scopo principale santificare sé stessi e gli altri. Così la famiglia costruita sul matrimonio sacramentale diventa una comunità santificante e mirante alla santità. Il matrimonio è un sacramento che chiama a dare una testimonianza all'amore sia sponsale, che creatore e corporeo (carnale) ${ }^{1}$. Basando su questa premessa sembra essere ragionevole chiedere: si può oggi parlare della dimensione mistica dell'unione corporea dei coniugi?

\section{RAGIONE DEL PROBLEMA SOPRAPPOSTO}

Attualmente la questione della dimensione mistica dell'unione corporea dei coniugi è diventata di nuovo un oggetto d'interesse dei teologi. A questo fanno un riferimento tra gli altri: A. Grün, K. Lubowicki, K. Knotz, K. Wojaczek e delle analisi scientifiche fatte dagli Istituti di studi sulla famiglia. Essa diventa qualche genere di risposta agli attacchi ideologici del mondo che cercano di creare attraverso l'individualizzazione dell'uomo una certa antitesi del personalismo cristiano ${ }^{2}$. In conseguenza cercano di arrivare al modo neomanicheismo di affrontare la questione, in cui la persona umana non sarebbe compresa in modo integrale, e la divisione sulla sfera carnale e spirituale causerebbe nell'uomo una deflagrazione pericolosa e negativa ${ }^{3}$.

Questa problematica però non è nuova. Poiché già nella corrente della mistica renana e cosiddetta mistica nuziale i vertici dell'unione con Dio si paragonava allo sposalizio. Questo è particolarmente visibile da san Giovanni della Croce che nel Cantico spirituale rassomiglia le tappe singole dello sviluppo della vita spirituale all'unione nell'amore degli amanti, i quali prima si cercano, poi incontrano, e finalmente si uniscono nell'amore ${ }^{4}$.

${ }^{1}$ Por. A. Sarmiento, Powotanie do świętości i matżeństwa. Homo Meditans XV. Małżeństwo - przymierze miłości, red. J. Misiurek, W. Słomka, Lublin 1995, s. 78-79.

${ }^{2}$ Więcej na ten temat zob.: S. Wielgus, Wobec wojującego sekularyzmu, Płock 2006; K. Wojaczek Matżeństwo - doświadczenie obdarowywania, Opole 2001; K. Lubowicki, Życie matżeńskie czynić eucharystiq, Kraków 2001.

${ }^{3}$ Temu sprzeciwiał się już papież Paweł VI w encyklice Humanie vitae 1968, nr 7-9.

\footnotetext{
${ }^{4}$ Por. Jan od Krzyża, Pieśń między duszq i Oblubieńcem, w: tenże, Dzieła. Pieśń
} 
Questo problema intraprendeva nei suoi scritti anche Karol Wojtyła. Nel libro Amore e responsabilità (Cracovia 1962) ha apprezzato il valore reale dell'istinto sessuale e $\mathrm{l}^{\prime}$ ha collocato nella prospettiva dell'amore compreso come un donarsi reciproco. Ha trattato a largo a tal scopo la questione del communio personarum. Poi, già come il Papa, ha ricordato che l'uomo è stato creato dal dono disinteressato dell'amore di Dio e può capire sé stesso solamente, se lui stesso diventerà un dono per gli altri. La somiglianza a Dio sta alla base della dignità dell'uomo e lo rende capace ad essere un dono. Nel centro del communio personarum nel matrimonio e nella famiglia sta dunque l'uomo come persona, perché soltanto egli sa regalare sé stesso e accettare il dono di un'altra persona, creando la comunità d'amore. Questa doppia capacità sottolinea il carattere personalistico della comunione delle persone ${ }^{5}$. Così questo communio personarum diventa un communio amoris di un reciproco donarsi dei coniugi, dunque ugualmente un dono completo di sé e un'affermazione del dono dell'altra persona. Questa reciprocità, che si realizza su tutti i livelli: spirituale, psicosomatica e emotiva, contribuisce alla partecipazione alla dimensione mistica dell'amore di Dio (cf. GS 24).

Contemporaneamente a questa tematica si è riferito nella sua prima enciclica il Papa Benedetto XVI. Vi ha distinto un amore incerto, cercante (dodim, ahaba) da un amore che è un superamento dell'egoismo nella direzione della scoperta di un altro uomo (agape). Il Papa ha proposto una definizione dell'amore, che è: "estasi non nel senso di un momento di ebbrezza, ma estasi come cammino, come esodo permanente dall'io chiuso in se stesso verso la sua liberazione nel dono di sé, e proprio così verso il ritrovamento di sé, anzi verso la scoperta di Dio..."6.

Dunque parlare sulla dimensione mistica dell'atto matrimoniale è comprensibile se si prende i coniugi nell'aspetto della comunione d'amore compreso come un reciproco donare e ritrovare sé stesso in questo dono.

duchowa, strofy 1-40, Kraków 1998, s. 525-531; H. Suzo, Księga Mądrości Przedwiecznej, wyd. polskie, tłum. W. Szymona, Poznań 1983; J. Tauler, Kazania, wyd. polskie, tłum. W. Szymona, Poznań 1985.

${ }^{5}$ Por. E. Kaczyński, Matżeństwo i rodzina jako komunia osób, Communio 36 (1986), nr 6, s. 6-8.

${ }^{6}$ Por. Benedykt XVI, Encyklika Deus Caritas est, Rzym 2005, nr 6. 


\section{SOTTO QUALE PUNTO DI VISTA ANALIZZARE LA QUESTIONE?}

Dopo aver affermato la ragione del problema messo bisogna porre una domanda successiva sull' aspetto della ricerca sulla questione. Essa concerne la dimensione mistica dell'unione corporea dei coniugi cristiani, dunque sarà esaminata nella corrente della mistica cristiana. È difficile definire, secondo J. Sudbrack, la questione della mistica. Egli crede che alla comprensione del fenomeno della mistica possono condurre quattro vie: psicologica, storico-sociologica, estetico-simbolica e religiosa. Sostiene che la mistica cristiana si pone in mezzo alla "tensione di due sfere", cioè: tra Dio e lo sperimentare di questo $\mathrm{Dio}^{7}$.

La sola idea di mystikos significa misterioso e deriva dalla tradizione ellenica. In una trasmissione diretta mostra a tutti una certa realtà misteriosa e inaccessibile. La sua comprensione esige una certa introduzione che spesso ha un carattere cultico. Tutte le grandi religioni hanno una loro mistica. Nella tradizione cristiana, secondo l'opinione di padre M. Chmielewski, essa concerne un triplo livello: biblico, sacramentale e spirituale $^{8}$. Proprio in una tale chiave sarà qui discussa la dimensione mistica dell'unione corporea dei coniugi.

Il livello biblico della mistica cristiana assume una tale lettura della Sacra Scrittura che permette di scoprire la realtà di Dio e la Sua opera di salvezza. Nel tema esaminato questo riguarda alcuni testi essenziali. Il primo è il brano dal Libro della Genesi, in cui Dio dopo la creazione dell'uomo e della donna ha detto a loro: "Siate fecondi e moltiplicatevi, riempite la terra; soggiogatela..." (Gen 1, 28). Con questo testo corrisponde un brano del vangelo secondo Matteo, in cui Gesù insegna: „Per questo l'uomo lascerà suo padre e sua madre e si unirà a sua moglie e i due saranno una carne sola. Così che non sono più due, ma una carne sola. Quello dunque che Dio ha congiunto, l'uomo non lo separi" $(19,5-6)$.

Un altro brano che si può ritenere essenziale nella discussione sull'espressione mistica della unione carnale dei coniugi è il testo del Libro di Tobia, nel quale i coniugi pregano prima della convivenza sessuale, offrendo a Dio il loro amore e chiedendo la benedizione per il loro vincolo

7 Por. Ch. Bernard, Teologia spirituale, Roma 1983, s. 457-460; J. Sudbrack, Mistyka. Doświadczenie własnego ja - doświadczenie kosmiczne - doświadczenie Boga, tłum. B. Białecki, Kraków 1996, s. 11-15.

${ }^{8}$ Por. M. Chmielewski, Mistyka, w: Leksykon duchowości katolickiej, Lublin-Kraków 2002, s. 536. 
matrimoniale, anche nell'aspetto carnale (cf. Tb 8, 4-9). L'atteggiamento di Tobia e Sara è, secondo Giovanni Paolo II, un paradigma dell'amore matrimoniale fondato sull'unità spirituale e carnale dell'uomo e della donna. Il mistero, che ha la sua sorgente in Dio, s'esprime attraverso la relazione carnale nel matrimonio sacramentale ${ }^{9}$.

Tobia e Sara pregano prima dell'unione carnale, riconoscendo la dipendenza della loro felicità da Dio, datore e Sorgente dell'amore. Così varcano il livello naturale elevandosi sul livello del matrimonio, compreso come mistero della presenza di Dio tra gli uomini. Vita matrimoniale, e in essa anche la convivenza sessuale diventa un rispettare la volontà di Dio e per questo, in qualche modo, un culto di Dio reso in un amore sponsale ${ }^{10}$.

Successivamente le parole dal libro del Cantico dei Cantici che anche si può giudicare chiave del tema delle basi bibliche della mistica cristiana rispetto alla comunità di vita matrimoniale, si concentrano sulla bellezza del corpo umano. Lo fanno non soltanto perché il solo corpo è affascinante, ma perché è un modo d'accettare un altro "io" personale, prendente l'iniziativa d'amore. Vivere la bellezza dell'amore abbraccia, secondo Giovanni Paolo II, tutto l'uomo. Il Cantico dei Cantici è un poema lirico di tematica amorosa. Un suo oggetto non è il matrimonio umano, ma mostrare la purezza dell'amore sponsale, nel quale soltanto si può scorgere i simboli del rapporto dello Sposo alla sposa, di Dio a Israele, di Gesù alla Chiesa ${ }^{11}$. Parla dell'amore perduto nel paradiso a causa del peccato dei primi genitori. Questo amore è stato ritrovato in Dio che ha chiamato uomo e donna a realizzarlo in vita matrimoniale. Attingendo dal Cantico dei Cantici Giovanni della Croce spiega gli stati mistici paragonando le tappe successive dello sviluppo della vita spirituale al fidanzamento e allo sposalizio mistico. Questo Cantico unisce perfettamente tutte le dimensioni dell'amore sponsale, mostrando il significato della sfera spirituale, psichica e fisica nel rapporto matrimoniale. Così affatto non deprezza niente, ma ristabilisce alla dimensione fisica dell'amore matrimoniale il suo luogo esatto.

Considerando il livello biblico della mistica cristiana non si può tralasciare l'insegnamento di san Paolo. Il suo insegnamento mostra il carattere religioso dell'istituzione del matrimonio nel Nuovo Testamento

${ }^{9}$ Por. Jan Paweł II, Mężczyzna i niewiasta stworzył ich, Watykan 1980, s. 27-28.

${ }^{10}$ Por. K. Knotz, Akt matżeński, Kraków 2001, s. 23-24.

11 Por. Wstęp do Pieśni nad Pieśniami, Biblia tysiąclecia, wyd. 3, Poznań 1991, s. 745-746. 
perché non soltanto colloca la mistica di vita matrimoniale sulla simbolicità dell'Antico Testamento dello sposalizio di Dio con Israele, ma allarga questa simbolicità al rapporto di Cristo con la Chiesa e con le chiese da lui fondate (cf. 2 Co 11, 2).

Essa è come un allargare e un aggiungere pratico all'insegnamento di Gesù sul tema dell'amore. Gesù si muove dentro le prospettive dell'amore proprio del Regno, non appellandosi alla struttura esistente del matrimonio. Esige l'amore e l'unione e l'uguaglianza tra donna e uomo. Successivamente la mistica cristiana secondo l'Apostolo delle Nazioni unisce i principi dell'amore matrimoniale con la cultura e i costumi d'allora. Il più eloquente testo, che mostra questa specificità della mistica matrimoniale di Paolo è la Lettera agli Efesini (cf. 5, 25-27.29-30)

In questo testo Paolo paragona la relazione di due persone al rapporto di Cristo con la Chiesa. La base della analogia così fatta è l'amore di Cristo per la Chiesa. Grazie a questo amore la sostanza del matrimonio è stata portata alla sfera dei valori soprannaturali, il ciò fa sì, che due persone non sono più uomini semplici, e tutto che fanno è soprannaturale (Ga 2, 20). Così tutti gli ideali matrimoniali realizzati in modo cristiano, in questo anche la convivenza sessuale, sono per i coniugi sorgente di un reciproco incanto, fortificando simultaneamente l'unita del matrimonio e la sua indissolubilità ${ }^{12}$.

L'aspetto sacramentale della mistica cristiana compreso come misterion dal greco, e dal latino sacramentum, prenderà i segni sacramentali, consentendo la partecipazione diretta alla vita divina. I coniugi aggregati spiritualmente e corporalmente diventano imitatori della presenza di Dio tra gli uomini. Il risultato del matrimonio è: la comunità d'amore, il vincolo cristiano dei coniugi, l'amore che contiene tutti gli elementi della persona umana e aspira alla unità dei due (cf. FC 13) ${ }^{13}$.

Così i coniugi cristiani che si amano diventano per loro stessi e per gli altri un segno della presenza viva di Dio tra loro. I coniugi sentendosi amati a vicenda sentono anche l'autenticità dell'amore di Dio e diventano i suoi trasmettitori nel mondo. Hanno l'influenza attiva su questo, se Cristo costantemente parteciperà alla loro vita matrimoniale e familiare (cf. GS 49) ${ }^{14}$.

12 Por. T. Goffi, Duchowość matżeńska, Kraków 2001, s. 31-34.

${ }_{13}$ Por. K. Majdański, Wspólnota życia i mitości, Poznań-Warszawa 1979; J. Bajda. Teologia miłości matżeńskiej, Ateneum Kapłańskie 67 (1967) z. 2, s. 175-186.

${ }^{14}$ Por. K. Knotz, Akt matżeński, s. 26-27. 
Il livello spirituale della mistica invece abbraccerà il processo della unione completa con Dio, che è lo scopo della vita spirituale del cristiano. Perciò bisogna far conoscere i modi d'intensificare la spiritualità matrimoniale. Si annovera tra di essi tra l'altro: la preghiera, la vita comune e l'unione carnale. La spiritualità matrimoniale secondo p. K. Knotz concerne l'incremento del legame matrimoniale e si realizza attraverso la sua celebrazione su: "l'altare della preghiera, del sedersi e del donarsi"15. L'ultimo concerne la convivenza sessuale che è una risposta dei coniugi alla volontà di Dio che li ha chiamati all'amore e lo ha assegnato a loro come un compito $($ Gen 1,28$)$. Così i coniugi sempre di più scoprono $1^{\prime}$ amore di Dio in tutte le dimensioni della loro vita: spirituale, psichica, emotiva e fisica.

Analisi della mistica da parte spirituale comprende l'atto matrimoniale come un certo genere del dono che i coniugi sacramentali portano a Dio, dividendo l'amore, che hanno ricevuto da Lui e che distribuiscono in loro e tra di loro, creando una comunione d'amore (cf. Tob 8, 4-8). Si tratta di sentire la vicinanza di Dio che può scorrere anche dalla sicurezza e fiducie alla persona amata. La vita autentica di sacramento del matrimonio sviluppa la mistica di vita matrimoniale nella Chiesa. Così mostra la presenza di Dio nella comunione matrimoniale e per di più in tutte le sue dimensioni.

La spiritualità matrimoniale, secondo Giovanni Paolo II, dovrebbe mirare a far risaltare la forza che consente l'autentica testimonianza cristiana di vita matrimoniale. Il Papa indica la sorgente di questa forza, basando sull'enciclica di Paolo VI Humanae vitae (1968). Accerta che questa fonte è l'amore innestato nel cuore dallo Spirito Santo (cf. Ro $5,5)$. Questo amore dovrebbe esprimersi in tutte le dimensioni di vita matrimoniale attraverso la realizzazione della vita cristiana sorta sul battesimo, e sviluppata e fortificata nel sacramento del matrimonio. Poiché questo sacramento dona ai coniugi la forza e in qualche modo è come se consacrasse loro, affinché possano fedelmente compiere le loro mansioni. Aiuta anche verso il perfezionamento della vita matrimoniale-familiare e nel donare la testimonianza cristiana in faccia al mondo. In tal modo il loro amore reciproco dinamizza intimamente il loro compito di cooperare e di penetrare l'amore di Dio, il datore di vita umana ${ }^{16}$.

${ }^{15}$ K. Knotz, Akt matżeński, s. 18-19.

16 Por. Paweł VI, HV 25; Jan Paweł II, Mężczyzna i niewiasta stworzyt ich, s. 126. 
La convivenza matrimoniale rispecchia la bellezza del vincolo spirituale che lega il marito e la moglie. Il corpo diventa la lingua di questa comunità $\mathrm{d}^{\prime}$ amore unita ${ }^{17}$. È proprio nella sfera spirituale che l'uomo decide e dirige i suoi comportamenti sessuali. Grazie a questo non tratta l'altra persona come un oggetto, ma come persona (cf. Ga 5, 13). Il vincolo sessuale s'approfondisce, quando la convivenza viene accompagnata dallo sviluppo spirituale. Del successo dell'atto matrimoniale decide l'anima che è una sorgente della dinamica sessuale dei coniugi ${ }^{18}$.

Lo scopo dello sviluppo della spiritualità matrimoniale è il riconoscere e l'attingere dall'amore matrimoniale sulla via alla santità dei coniugi, e l'amore fisico non è più un ostacolo, ma un modo d'esprimere il legame del matrimonio sacramentale. L'essenza della unione matrimoniale è il donarsi a vicenda ${ }^{19}$. Questa è la mistica dell'unione dei coniugi. Essa si realizza anche nella dimensione carnale. Evidentemente questa sfera di vita matrimoniale non è stata sviluppata nella Sacra Famiglia, ma cambiata nel dono completo dei coniugi per Dio che permette di realizzare il communio amoris e cioè cosidetti matrimoni bianchi che realizzano l'amore reciproco nella temperanza completa, così come nel caso della Sacra Famiglia di Nazareth. La convivenza matrimoniale dunque non è d'ostacolo verso la santità, se rispetta la libertà e la dignità dell'amore dei coniugi. Anzi la donazione mutua permette di partecipare alla dimensione mistica della comunità $\mathrm{d}^{\prime}$ amore $^{20}$.

\section{QUALE È IL MODO PER PRESENTARE LA TEOLOGIA DEL CORPO NELL'UNIONE DEI CONIUGI?}

Dopo aver trattato sulla dimensione mistica dell'unione matrimoniale sotto l'aspetto biblico, sacramentale e spirituale, rimane ancora dare uno sguardo alla parte psico-fisica. È stato detto sopra che i coniugi celebrano il sacramento del matrimonio anche nell'atto dell'unione carnale. Come dunque guardare sul corpo, se in qualche modo esso diventa materia del sacramento?

\footnotetext{
17 Por. Z. Nosowski, Parami do nieba, s. 163-164.

${ }_{18}$ Por. M. Laroche, Maty Kościót, Poznań 1989, s. 88.

19 Por. tamże, s. 22.

${ }^{20}$ Por. A. Sobczyk, Communio caritatis, s. 280-289.
} 
La teologia contemporanea sottolinea il significato del corpo umano come l'unità con l'anima anche nell'aspetto sessuale. Avverte di non trattarlo in modo strumentale, ma complementare nella persona. Esso partecipa pure nel vivere Dio durante l'amore matrimoniale (cf. GS 14) ${ }^{21}$. Nel corpo umano si estrinseca la forza Divina e realizza la cooperazione nell'atto creatore. Il dono del corpo legato al dono di tutta la persona, integra la comunione dell'amore matrimoniale basato sul donarsi complessivo, reciproco.

L'uomo è stato creato all'immagine e somiglianza di Dio (cf. Gen 1, 27). L'uomo e la donna, sono stati chiamati a far sì che nel dono di sé possano di nuovo offrire loro stessi a vicenda. Secondo Giovanni Paolo II la carnalità non è un oggetto del dono nell'atto matrimoniale, ma essa stessa è il dono della persona alla persona. Non è soltanto un segno da regalare, ma anche un segno d'accettare il dono, costituente il communio personarum. Questo fa crescere l'amore reciproco e la prontezza alla creazione del communio amoris ${ }^{22}$. Inoltre la lingua del corpo in qualche modo interviene nell'atto matrimoniale tra questo, che è biologico, e questo che è spirituale ${ }^{23}$.

Discutendo sulla teologia del corpo vale la pena domandare sugli scopi principali della unione carnale dei coniugi. Secondo il Cardinale Wojtyła il più importante di essi è l'amore sponsale - la dedizione - l'amore legato al desiderio di un reciproco offrirsi delle persone ${ }^{24}$.

L'amore dell'uomo e della donna diviene arricchita dal dono della grazia e dell'amore sponsale di Cristo alla Chiesa. Conforme all'insegnamento conciliare: "Questo amore è espresso e sviluppato in maniera tutta particolare dall'esercizio degli atti che sono propri del matrimonio. Ne consegue che gli atti coi quali i coniugi si uniscono in casta intimità sono onesti e degni; compiuti in modo veramente umano, favoriscono la mutua donazione che essi significano ed arricchiscono vicendevolmente nella gioia e nella gratitudine gli sposi stessi. Quest'amore, ratificato da un impegno mutuo e soprattutto consacrato da un sacramento di Cristo,

${ }^{21}$ Por. W. Gasidło, Z zagadnień etyki matżeńskiej i rodzinnej, Kraków 1994, s. $147-148$.

${ }^{22}$ Por. Jan Paweł II, Mężczyzna i niewiasta, s. 32-33; T. Styczeń, Ciało jako znak obrazu Stwórcy, w: tenże (red.), Mężczyzna i niewiasta stworzyt ich. Chrystus odwotuje się do początku, Lublin 1981, s. 89; 109-110.

${ }^{23}$ Por. R. Buttiglione, Dar ciała darem osoby, w: P. Ślęczka (red.), Dar ciała darem osoby, Lublin 2005, s. 87.

${ }^{24}$ Por. K. Wojtyła, Miłość i odpowiedzialność, Kraków 1962, s. 84-85. 
resta indissolubilmente fedele nella prospera e cattiva sorte..." (GS 49). La penetrazione reciproca dei fattori del divino e umano nell'amore matrimoniale conduce loro a un donarsi reciproco. In questo modo l'abituale communio personarum diventa il communio amoris.

L'amore dei coniugi è una partecipazione all'amore di Dio. Contenuto di questa partecipazione sono ugualmente gli elementi fisici, la sfera psichica e spirituale. Bisogna oggi, in confronto ai sintomi della svalutazione dell'amore o del suo improprio identificare con i comportamenti edonistici o con il trattamento oggettivo dell'altro, decisamente sottolineare che l'intimo incontro dei coniugi non può essere identificato con l'amore, ma è un suo segno e modo di trasmettere, esprimere dell'amore sponsale, per il quale diventano dono vicendevole ${ }^{25}$. Soltanto in un tale aspetto si può esaminare la dimensione mistica della unione carnale dei coniugi.

Uno scopo successivo dell'atto sessuale è il consolidamento dell'unione dei due. Un vivere insieme del marito e della moglie nell'unione carnale diretta all'amore sponsale serve alla comunione e l'unità. L'uomo e la donna si completano a vicenda creando la comunione delle persone appoggiata sull'amore sponsale (cf. GS 7)

Infine un'altra tendenza essenziale dell'unione matrimoniale è la procreazione - che secondo Gen 1,28 è una missione principale dei coniugi (cf. GS 50; CCC 2367).

Non si può anche tralasciare la partecipazione della sfera psichica all'unione carnale dei coniugi. Attraverso la lingua del corpo i coniugi entrano nella sfera psichica delle loro personalità. L'atto sessuale privo della sfera psichica condurrebbe a rendere come un oggetto l'unione delle persone. La sfera psichica insieme con la coscienza, l'intelletto sono un suo elemento integrale. A questo si aggiunge affettività, e grazie ad essa il provare del piacere, il desiderio reciproco l'uno dell'altro, la nostalgia e la tenerezza. Grazie ad esse le persone aspirano a un offrirsi reciproco ${ }^{26}$. La condizione di vivere pienamente l'atto sessuale nell'aspetto psichico è la maturità personale dell'uomo e della donna insieme con la responsabilità per sé e per l'altra persona ${ }^{27}$.

${ }^{25}$ Por. Jan Paweł II, Mężczyznq i niewiasta, s. 61.

${ }^{26}$ Por. E. Sujak, Kontakt psychiczny w matżeństwie, Katowice 1971, s. 114.

${ }_{27}$ Por. W. Półtawska, Przygotowanie do matżeństwa, Kraków 1993, s. 44-45. 


\section{IN CHE COSA CONSISTE LA MISTICA DI UN INCONTRO MATRIMONIALE INTIMO?}

Dopo aver riflettuto la ragione del problema messo e aver presentato l'aspetto di ricerca, aver discusso sulla partecipazione delle facoltà singolari dell'uomo e del suo corpo nell'atto della unione matrimoniale, si può rispondere sulla domanda: in che cosa consiste la mistica di un intimo incontro dei coniugi. Con W. Fijałkowski bisogna accertare che, in un significato più largo, la dimensione mistica dell'unione corporea dei coniugi consiste nell'incontrare Dio in un'altra persona. Questo incontro, in questo caso - l'atto matrimoniale, è un modo di comunicare e un modo di esprimere il suo dono all'altra persona. Così i coniugi entrano nel dialogo che svela a loro l'un l'altro. Varcano i confini carnali, per trasmettere loro stessi nella sfera di tutta la loro personalità ${ }^{28}$.

Però la stessa convivenza deve condurre all'unione con Dio nell'amore. In questo consiste il senso esatto della dimensione mistica dell'unione carnale dei coniugi. Si deve qui rivolgere alle esperienze dei mistici, secondo cui, sul livello superiore del rapporto con Dio, ci si arriva alla sublimazione delle emozioni nell'amore spirituale al coniuge e a Dio. Così, come dice A. Grün, migliora „la qualità" d'amare Dio e l'altro uomo ${ }^{29}$.

T. Goffi nel suo libro „Spiritualità matrimoniale” (Cracovia 2001), sostiene che nell'unione carnale dei coniugi cristiani è presente la grazia dello Spirito di Cristo, che si rende presente in questo atto attraverso la forza della carità. Così l'unione carnale dei coniugi passa tramite Gesù, creando io-Egli-tu. Questo non diminuisce l'immediatezza del rapporto dei coniugi, ma edifica sulla presenza dello Spirito di Cristo in essa. La presenza della terza Persona della Trinità è un grande arricchimento di questo amore ${ }^{30}$.

Questo incontro conduce a un migliore conoscere l'un l'altro. Un tale atto contribuisce ad approfondire la propria persona e la persona del o della coniuge. Durante l'unione carnale i coniugi si rendono conto che il dono reciproco fatto dal corpo è un segno della dedizione di tutta la persona, è un tocco della propria identità e l'esistenza (cf. FC 80) ${ }^{31}$.

${ }^{28}$ Por. H. Łuczak, Dorastanie do miłości, Warszawa 1990, s. 112.

29 Por. A. Grün, G. Redl, Mistyka i eros, Kraków 1998, s. 32.

${ }^{30}$ Por. T. Goffi, Duchowość matżeńska, s. 159.

${ }^{31}$ Por. R. Buttiglione, Dar ciata darem osoby, s. 85-86. 
L'unione carnale è anche la soglia per varcare lo spazio sensuale. M. Laroche crede che dopo la realizzazione dei compiti dell'atto matrimoniale i coniugi possono varcare la soglia dell'esperienza religiosa, che indica l'occasione d'incontrare Dio in questo momento.

Questo è possibile, perché nell'atto carnale s'esprimono anche le facoltà spirituali, nei quali ci si arriva all'incontrare Dio. La presenza del Creatore in questo atto è eccezionale. L'unione matrimoniale deve essere un incontro nel nome di Gesù, a cui i coniugi appartengono. Non possono dunque disporre col corpo diversamente se non nello spirito di Cristo e per le Sue opere (cf. 1 Co 6, 15-20) . $^{32}$

L'atto matrimoniale come una cooperazione nell'opera creatrice di Dio si può interpretare come il culto reso al Signore. L'intimo incontro dei coniugi diventa la base, sulla quale si costruisce un segno sacramentale del matrimonio. Il matrimonio stesso invece è un atto della venerazione di Dio in Gesù e nella Chiesa. Trasmettendo l'amore l'un l'altro nell'atto matrimoniale i coniugi rendono gloria a Dio che li ha creati nell'amore, ha elargito a loro l'amore e li ha chiamati all'amore (cf. FC 40) ${ }^{33}$. Perciò i coniugi dovrebbero accettare la loro sessualità come un incoraggiamento ad un più profondo rivolgersi a Dio. L'amore carnale ha un'influenza essenziale sul costruire della comunione delle persone.

A questo si riferisce W. Fijałkowski che crede che Dio entrando nell'amore matrimoniale solleva l'atto matrimoniale sul livello superiore, preparando già i coniugi all'amore eterno ${ }^{34}$.

Parlando della mistica dimensione dell'unione carnale dei coniugi non si può tralasciare il tema della temperanza sessuale che nell'amore matrimoniale ha un ruolo significativo. Questa è una preparazione naturale ad un incontro sessuale, e dall'altro lato difende contro l'egoismo e l'autosufficienza. Permette, ne fanno attenzione gli psicologi e i sessuologi, di conservare la freschezza dell'amore e di mantenere i suoi affetti. Perciò i coniugi dovrebbero nei rapporti intimi decidersi di mantenere periodicamente la temperanza, perché i rapporti primitivi, adempiuti senza limiti sotto l'influenza di soli stimoli, portano all'esaurimento e alla noia ${ }^{35}$.

32 Por. Paweł VI, Rodzina szkoła świętości, Ateneum Kapłańskie 396 (1975), nr 1, s. 8.

33 Por. Jan Paweł II, Mężczyzna i niewiasta, s. 448.

${ }^{34}$ Por. W. Fijałkowski, Miłość w spotkaniu płci, Warszawa 1977, s. 92; W. Słomka Matżeństwo - duchowość w cielesności, Homo meditans, t. XV: Matżeństwo - przymierze mitości, red. J. Misiurek, W. Słomka, Lublin 1995, s. 57-69.

${ }^{35}$ Por. E. Sujak i inni. 
È difficile interpretare la temperanza nella luce dell'aridità mistica, conosciuta nel campo della teologia di spiritualità, nella quale Dio sembra all'anima lontano, ma l'anima sa che Lo incontrerà presto; però questo è il tempo d'attesa all'unione con la persona amata. L'essenza della esperienza mistica consiste nello sperimentare Dio così come Egli è in sé stesso, e nella cosciente partecipazione al Suo amore ${ }^{36}$.

Il significato più profondo della temperanza spiegano nel loro insegnamento i Papi: Paolo VI e Giovanni Paolo II. Secondo la loro opinione la temperanza diventa un'essenziale condizione "della lingua del corpo" e della sottomissione reciproca nel timore di Cristo. La virtù della temperanza non si limita però soltanto a mettere la resistenza alla concupiscenza, ma per questa resistenza apre ai valori più profondi e più maturi che s'uniscono con il significato sponsale del corpo umano e con la libertà del dono di sé ${ }^{37}$.

Riassumendo bisogna notare che nella spiritualità cristiana da tanti secoli si parlava della dimensione mistica dell'unione carnale dei coniugi. Questo tema è ritornato come una risposta in confronto al secolarismo belligerante e all'ideologia del neomanicheismo, che è un pericolo per il personalismo cristiano. Da qui deriva l'interesse così vivo per questo problema e vengono create delle basi filosofiche e teologiche, particolarmente nell'insegnamento di Giovanni Paolo II e di teologi odierni. Alla base delle loro ricerche si può sottolineare il significato dell'unione carnale dei coniugi per lo sviluppo dell'amore matrimoniale e verso Dio. In modo migliore il carattere mistico dell'unione carnale dei coniugi si può leggere nell'aspetto biblico, sacramentale e spirituale. In questo modo l'amore matrimoniale che si realizza nell'atto sessuale diventa il compimento della volontà di Dio, il compimento dell'amore nella dimensione verticale e orizzontale, come pure un segno della presenza di Dio e dell'unione con Lui in un'altra persona.

Abstract. Mystical dimension of bodily uniting spouses.To sum up one should notice that they in the Christian spirituality foe many centuries talked about the mystical dimension of bodily uniting spouses. Subject tem returned as the e reply towards the fighting sexuality and the ideology of neomaniceism in danger for the Christian personalism. Hence this way the keen interest in this issue and creating

36 Por. S. Urbański, Fenomen mistyki, Życie Duchowe, 43(2005), nr 2, s. 80.

${ }^{37}$ Por. Paweł VI, Encyklika Humanae Vitae, nr 21; Jan Paweł II, Mężczyzną i niewiastą, s. 131-132. 
philosophical and theological bases for it, particularly in the teaching John Paul II and of the contemporary theologians. Based on their examinations it is possible to emphasize the significance of bodily uniting spouses for the development of married love and towards God. Best to read out mystical character of bodily uniting spouses in the biblical, sacramental, and spiritual aspect. In this way married love finding fulfillment in the sexual act is becoming a filing of the will of God, with filling of love in the vertical and horizontal dimension as well as with sing of the presence of God and uniting withhim) God in the second man.

Key words: Mystic, conjugal act, sanctity

Streszczenie.Mistyczny wymiar zjednoczenia cielesnego małżonków chrześcijańskich. W duchowości chrześcijańskiej od wielu wieków mówiło się o mistycznym wymiarze zjednoczenia cielesnego małżonków. Temat ten powrócił jako odpowiedź wobec wojującego sekularyzmu i ideologii neomanichejskiej, będącej zagrożeniem dla chrześcijańskiego personalizmu. Stąd tak żywe zainteresowanie tym zagadnieniem i stworzenie mu filozoficznych i teologicznych podstaw, szczególnie w nauczaniu Jana Pawła II i teologów współczesnych. Na podstawie ich badań można podkreślić znaczenie zjednoczenia cielesnego małżonków dla rozwoju miłości małżeńskiej i względem Boga. Mistyczny charakter zjednoczenia cielesnego małżonków najlepiej odczytać w aspekcie biblijnym, sakramentalnym i duchowym. W ten sposób miłość małżeńska realizująca się w akcie seksualnym staje się wypełnieniem woli Bożej, wypełnieniem miłości w wymiarze wertykalnym i horyzontalnym, jak również znakiem obecności Boga i zjednoczeniem z Nim w drugim człowieku.

Słowa kluczowe: Mistyka, akt małżeński, świętość 